\title{
Application of Reassigned Wavelet Scalogram in Wind Turbine Planetary Gearbox Fault Diagnosis under Nonstationary Conditions
}

\author{
Xiaowang Chen and Zhipeng Feng \\ School of Mechanical Engineering, University of Science and Technology Beijing, Beijing 100083, China \\ Correspondence should be addressed to Zhipeng Feng; fengzp@ustb.edu.cn
}

Received 27 June 2015; Accepted 31 August 2015

Academic Editor: Chuan Li

Copyright @ $2016 \mathrm{X}$. Chen and Z. Feng. This is an open access article distributed under the Creative Commons Attribution License, which permits unrestricted use, distribution, and reproduction in any medium, provided the original work is properly cited.

Wind turbine planetary gearboxes often run under nonstationary conditions due to volatile wind conditions, thus resulting in nonstationary vibration signals. Time-frequency analysis gives insight into the structure of an arbitrary nonstationary signal in joint time-frequency domain, but conventional time-frequency representations suffer from either time-frequency smearing or crossterm interferences. Reassigned wavelet scalogram has merits of fine time-frequency resolution and cross-term free nature but has very limited applications in machinery fault diagnosis. In this paper, we use reassigned wavelet scalogram to extract fault feature from wind turbine planetary gearbox vibration signals. Both experimental and in situ vibration signals are used to evaluate the effectiveness of reassigned wavelet scalogram in fault diagnosis of wind turbine planetary gearbox. For experimental evaluation, the gear characteristic instantaneous frequency curves on time-frequency plane are clearly pinpointed in both local and distributed sun gear fault cases. For in situ evaluation, the periodical impulses due to planet gear fault are also clearly identified. The results verify the feasibility and effectiveness of reassigned wavelet scalogram in planetary gearbox fault diagnosis under nonstationary conditions.

\section{Introduction}

Wind turbines are playing an increasingly significant role in energy strategy. However, harsh working conditions, for example, wind gust, dust, and unpredictable heavy load, make the power transmission system prone to fault, which may lead to catastrophic breakdown or even productivity and economic losses. Planetary gearbox is one of the key components of wind turbine powertrain, for its merits of high gear transmission ratio and large load bearing capacity in a compact structure. Therefore, planetary gearbox fault diagnosis has been an important topic and has drawn increasing attentions recently [1-3].

Planetary gearbox exhibits quite unique dynamic behavior due to its special transmission structure and kinetics. McFadden [4] recognized that the varying phase angle of the planet gear vibration is responsible for spectra asymmetry. Inalpolat and Kahraman [5, 6] investigated the mechanisms of sideband harmonics in the vicinity of meshing frequency and its integer multiples using a general model and further developed a dynamic model to predict modulation sidebands due to manufacturing errors. Feng and Zuo [7] proposed planetary gearbox vibration signal models and deprived equations to calculate both local and distributed fault frequencies. These researches show that planetary gearbox vibration signals feature complexity and modulation and therefore have quite different spectral characteristics from that of fixed-shaft gearboxes.

In order to extract potential fault feature from planetary gearbox vibration signals, researchers proposed different methods. McNames [8] used continuous-time Fourier series (CTFS) analysis to invest the frequency characteristics. Chen et al. [9] applied ensemble multiwavelet transform with adaptive multiwavelet basis function to extract weak fault feature of a planetary gearbox. Lei et al. [10] proposed an adaptive stochastic resonance method to extract weak fault feature characteristics from noisy vibration signals of planetary gearbox. Considering the spectral complexity of 
planetary gearbox vibration signals, Feng et al. [11] presented a joint amplitude and frequency demodulation analysis based on Teager energy operator and ensemble empirical mode decomposition (EEMD) to diagnose planetary gearbox fault. Barszcz and Randall [12] employed the spectral kurtosis to detect gear tooth crack in a wind turbine planetary gearbox. Sun et al. [13] customized the multiwavelets based on the redundant symmetric lifting scheme to detect the incipient pitting faults in planetary gearbox. Li et al. [14] extracted the fault characteristics frequencies of planetary gearbox based on empirical mode decomposition and adaptive multiscale morphological gradient filter.

Most of above works were based on an assumption of signal stationarity, that is, constant running speed and load. However, in real-world wind turbine applications, volatile wind conditions result in nonstationary vibration signals. For example, the running speed varies with respect to the unpredictable wind power, and transient strong wind may lead to a shock. Under nonstationary conditions, planetary gearbox vibration signals always feature intricate frequency component structure $[15,16]$, manifesting as time-varying amplitude modulation (AM) and frequency modulation (FM). Due to the nonstationary nature, conventional Fourier transform most likely fails to thoroughly reveal the true frequency structure. To the best of our knowledge, the literature reported on planetary gearbox fault diagnosis under nonstationary conditions has been very limited [17]. Bartelmus and Zimroz [18] proposed an indicator that reflects the linear dependence between the meshing frequency amplitude and the operating condition for condition monitoring of planetary gearboxes. Chaari et al. [19] developed a mathematical model to understand the dynamic behavior of planetary gear under variable load condition. Yang and Zhang [20] studied the running characteristics of wind turbine planetary gearbox considering time-varying speed and load.

Time-frequency analysis (TFA) is capable of revealing time-frequency structure despite time variation of frequency components and thus is suitable for analyzing nonstationary signals [21]. Linear time-frequency analysis methods like short time Fourier transform (STFT) and continuous wavelet transform (CWT) essentially represent signals by weighted sum of a series of bases localized in both time and frequency domains. But their time-frequency resolution is governed by Heisenberg uncertainty principle and resolution in one domain can only be improved at expense of the other, resulting in time-frequency smearing [22]. Bilinear time-frequency distribution presents the signal energy on time-frequency plane $[23,24]$. The Wigner-Ville distribution (WVD) is the basis of almost all bilinear TFA methods, with merit of finest time-frequency resolution. However, it is subjected to inevitable cross-term interferences when analyzing multicomponent signals. Even for monocomponent signals, it still suffers from inner interference if the IF curve is nonlinear. Although improved methods, smoothed pseudo Wigner-Ville distribution (SPWVD), for example, can effectively suppress the cross-term interferences, they in turn deteriorate time-frequency resolution.

Fortunately, reassigned wavelet scalogram has merits of excellent time-frequency resolution and good suppression of cross-terms and thus can clearly exhibit the time-frequency structure of nonstationary signals. However, its applications in machinery fault diagnosis have been very limited, and only a few published works focus on this topic. Peng et al. [25] studied the characteristics of rotor-stator rubbing impacts and applied both scalogram and reassigned scalogram for impact detection. Ma et al. [26] summarized the symptoms of rotor-stator rubbing fault based on reassigned scalogram.

In this paper we further extend the application of reassigned wavelet scalogram to planetary gearbox fault diagnosis under nonstationary conditions [27] and validate its effectiveness in extracting gear fault features. The remainder of this paper is structured as follows. In Section 2, the detailed algorithm of scalogram and reassigned scalogram is introduced. In Section 3, a numerical simulated nonstationary signal encompassing a time-varying frequency and periodical impulses is analyzed to illustrate the performance of reassigned scalogram. In Sections 4 and 5, the effectiveness of reassigned scalogram is further validated by analysis of lab experimental planetary gearbox vibration signals in both local and distributed sun gear fault cases, as well as in situ wind turbine planetary gearbox vibration signals measured from a wind farm. Finally, conclusions are drawn in Section 6.

\section{Reassigned Wavelet Scalogram}

2.1. Continuous Wavelet Transform. Wavelet transform (WT) has advantage of better time-frequency resolution over short time Fourier transform (STFT). Rather than using a constant window function for whole time-frequency plane analysis, it employs different window functions for analyzing different frequency bands of the signal. Therefore, WT is popular among linear TFA methods.

For an arbitrary finite-energy signal $x(t) \in L^{2}(R)$, its CWT is defined as

$$
\begin{aligned}
W_{x}(a, b ; \psi) & =\left\langle x(t), \psi_{a, b}(t)\right\rangle \\
& =a^{-1 / 2} \int_{-\infty}^{\infty} x(t) \psi_{a, b}^{*}(t) d t,
\end{aligned}
$$

where asterisk $*$ stands for complex conjugate, $a>0, a$ and $b$, respectively, represent the scale and time offset, and $\psi_{a, b}(t)$ is the family of wavelets generated by dilation and translation from the mother wavelet $\psi(t)$ :

$$
\psi_{a, b}(t)=a^{-1 / 2} \psi\left(\frac{t-b}{a}\right) .
$$

Morlet wavelet is most commonly used in CWT, and it is defined as

$$
\psi(t)=\pi^{-1 / 4}\left(e^{-i \omega_{0} t}-e^{-\omega_{0}^{2} / 2}\right) e^{-t^{2} / 2} .
$$

The wavelet scalogram is defined as

$$
S_{x}(a, b ; \psi)=\left|W_{x}(a, b ; \psi)\right|^{2} .
$$

2.2. Reassigned Wavelet Scalogram. Although wavelet scalogram can reveal the constituent frequency components of 


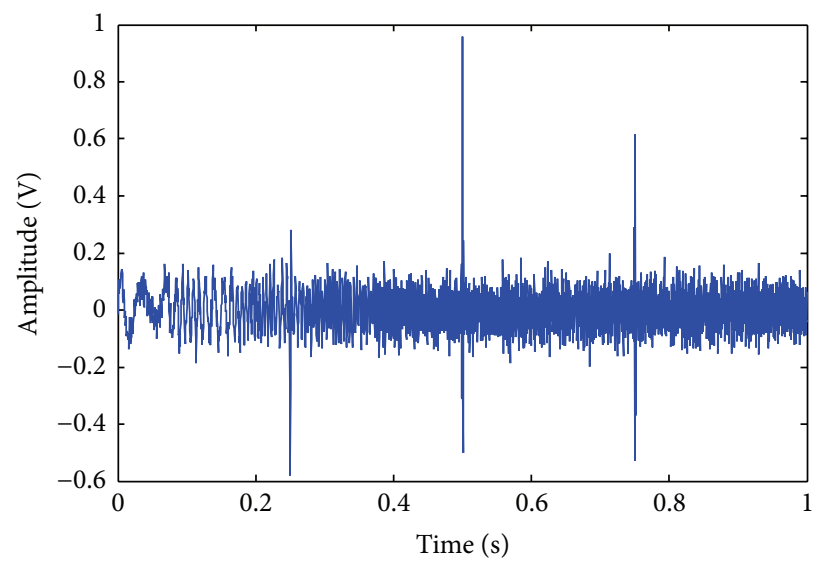

(a) Waveform

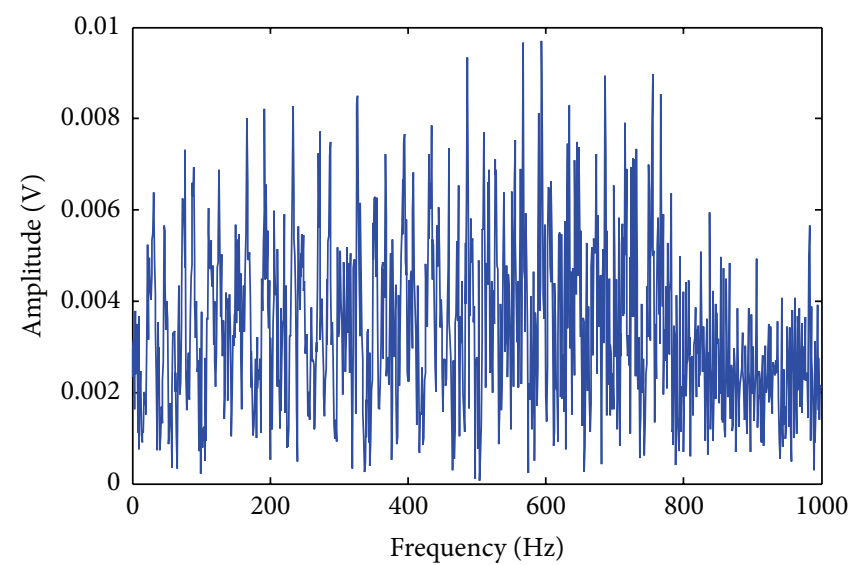

(b) Fourier spectrum

FIGURE 1: Simulated signal.

a signal and show how the energy of each component varies with time and frequency, its time-frequency resolution is still subject to the Heisenberg-Gabor inequality; that is, fine time localization and frequency resolution can hardly be obtained simultaneously. For example, it has fine time localization but coarse frequency resolution in high-frequency regions, while it has coarse time localization but fine frequency resolution in low-frequency regions. Therefore, for nonstationary signals with instantaneous frequency curves changing in a wide frequency region, the time-frequency blurring phenomenon may arise, thus hindering an accurate fault feature extraction.

In order to improve the readability of conventional wavelet scalograms, Auger and Flandrin [28] proposed the reassignment method. The wavelet scalogram $S_{x}(a, b ; \psi)$ does not represent the signal energy at the location $(a, b)$ on the time-scale/frequency plane but the mean energy in the vicinity of location $(a, b)$. Therefore, it is reasonable to reallocate the signal energy $S_{x}(a, b ; \psi)$ to the center of gravity in the vicinity of location $(a, b)$. The new coordinates become

$$
\begin{aligned}
& \frac{\omega_{0}}{a^{\prime}(b, a)} \\
& \quad=\frac{\omega_{0}}{a}+\operatorname{Im}\left\{\frac{\mathrm{CWT}_{x}(a, b ; \widehat{\psi}) \mathrm{CWT}_{x}^{*}(a, b ; \psi)}{2 \pi a\left|\mathrm{CWT}_{x}(a, b ; \psi)\right|^{2}}\right\}, \\
& b^{\prime}(a, b) \\
& \quad=b-\operatorname{Re}\left\{a \frac{\mathrm{CWT}_{x}\left(a, b ; \psi^{\prime}\right) \mathrm{CWT}_{x}^{*}(a, b ; \psi)}{\left|\mathrm{CWT}_{x}(a, b ; \psi)\right|^{2}}\right\},
\end{aligned}
$$

where

$$
\begin{aligned}
\psi^{\prime}(t) & =t \psi(t), \\
\widehat{\psi}(t) & =\frac{d \psi}{d t}(t) .
\end{aligned}
$$

Thus the reassigned wavelet scalogram is cast as

$$
\begin{aligned}
& R S_{x}\left(a^{\prime}, b^{\prime}\right)=\int_{-\infty}^{+\infty} \int_{-\infty}^{+\infty}\left(\frac{\widehat{a}}{a}\right)^{2} S_{x}(a, b) \delta\left[\widehat{b}-b^{\prime}(a, b)\right] \\
& \cdot \delta\left[\widehat{a}-a^{\prime}(a, b)\right] d a d b .
\end{aligned}
$$

\section{Simulation Evaluation}

In this section, we examine the performance of reassigned wavelet scalogram in identifying time-varying frequency components and transient impulses characteristic of typical gear fault under nonstationary conditions. A numerical simulated signal is generated:

$$
\begin{aligned}
& s(t)=A \sin \left(2 \pi(\int_{\text {chirp }} 800 t+\underbrace{\int 50 \cos (2 \pi 10 t)}_{\mathrm{FM}})\right) \\
& \quad+\underset{\text { impulse }}{\delta(t)+n(t),}
\end{aligned}
$$

where $t=0: 0.0005: 1 \mathrm{~s}, A=0.1$ is the amplitude of timevarying frequency component, and $\delta(t)$ stands for impulses at time instant of $0.25,0.5$, and $0.75 \mathrm{~s}$. To mimic background noise, a Gaussian white noise $n(t)$ at signal to noise ratio (SNR) of $8 \mathrm{~dB}$ is added. This signal encompasses three nonstationary conditions, that is, linear frequency modulation (chirp), frequency fluctuations (FM), and periodical impulses. Its waveform and Fourier spectrum are plotted in Figures 1(a) and 1(b).

From the signal waveform, Figure 1(a), we can clearly see the time instant of the three impulses but cannot identify the period of time-varying frequency components. Neither can we do from the Fourier spectrum, Figure 1(b), due to the nonstationarity of the simulated signal.

Figure 2 shows the time-frequency analysis results of the signal. In the wavelet scalogram, Figure 2(a), the major signal components and their time variation are roughly 


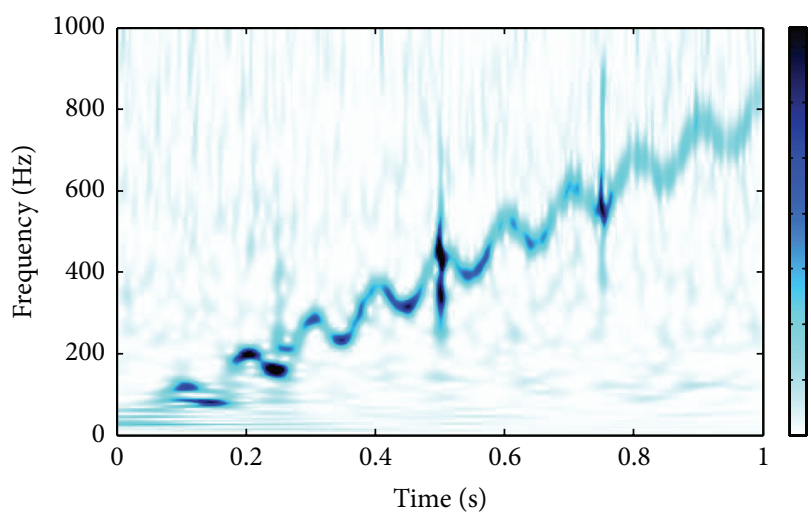

(a) Wavelet scalogram

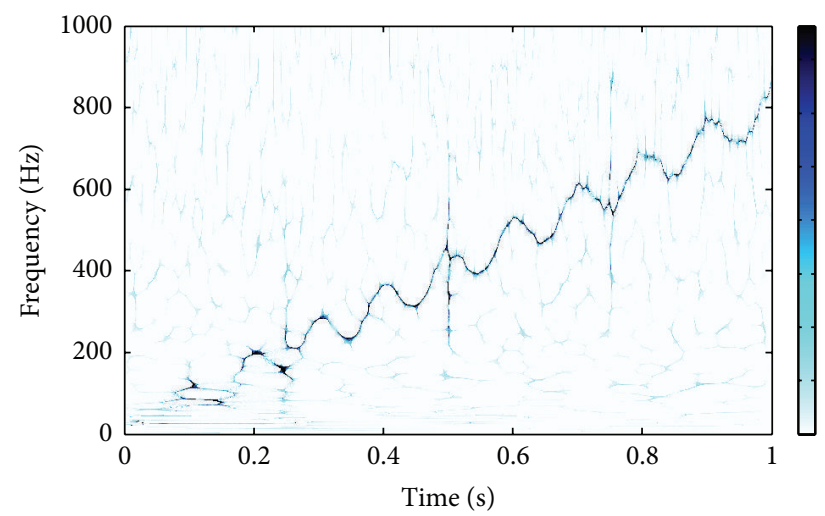

(b) Reassigned scalogram

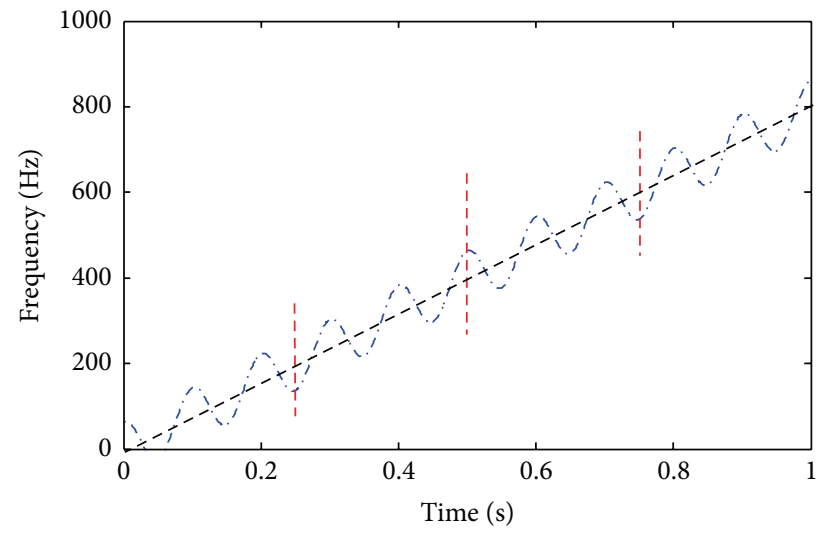

(c) True frequency constitution

FIGURE 2: TFRs of the simulated signal.

shown: a nearly sinusoidal fluctuating frequency rides on a linear trend, together with three impulses. However, timefrequency blurring occurs because the instantaneous frequency of the time-varying frequency component is nonlinear. This results in a lower time-frequency resolution and may hinder an accurate time-frequency localization of each signal component.

In the reassigned scalogram, Figure 2(b), both the timevarying frequency component and the three impulses are clearly displayed, owing to the much better time-frequency resolution. More importantly, the identified instantaneous frequency curve of the time-varying component and the time-frequency distribution of the three impulses match well with the true time-frequency constitution shown in Figure 2(c). This result demonstrates the capability of reassigned scalogram in identifying both time-varying frequency component and transient impulses. Therefore, reassigned scalogram has the potential to diagnose planetary gearbox fault under nonstationary conditions.

\section{Experimental Evaluations}

Experiments were carried out on a wind turbine drivetrain test rig in University of Ottawa lab, to examine the effectiveness of reassigned wavelet scalogram on real-world planetary gearbox fault diagnosis.
TABLE 1: Fixed-shaft gearbox configuration.

\begin{tabular}{lcc}
\hline Gear & \multicolumn{2}{c}{ Number of gear teeth } \\
& Stage 1 & Stage 2 \\
\hline Input & 32 & - \\
Intermediate & 80 & 40 \\
Output & - & 72 \\
\hline
\end{tabular}

4.1. Experimental Settings. In order to simulate the working condition of real-world planetary gearbox in wind farms, a wind turbine drivetrain composed of a two-stage planetary gearbox and a two-stage fixed-shaft gearbox is set up, as shown in Figure 3. The power flow path is as follows: drive motor $\rightarrow$ fixed-shaft gearbox $\rightarrow$ planetary gearbox $\rightarrow$ main shaft $\rightarrow$ magnetic powder brake. The configuration parameters of the two gearboxes are listed in Tables 1 and 2, respectively.

Accelerometers are mounted on the top of planetary gearbox casing to measure planetary gearbox vibration signal at a sampling frequency of $20,000 \mathrm{~Hz}$. A load of $16.3 \mathrm{~N} \cdot \mathrm{m}$ is applied at the output end of planetary gearbox stage 2 by loading device. In consideration of the wind turbine blade rated speed of $25 \mathrm{rpm}$, that is, $0.417 \mathrm{~Hz}$, we collect the vibration data during specific speed-down processes. 
TABLE 2: Planetary gearbox configuration.

\begin{tabular}{lcc}
\hline Gear & \multicolumn{2}{c}{ Number of gear teeth } \\
& Stage 1 & Stage 2 \\
\hline Ring & 100 & 100 \\
Planet & $40(4)$ & $36(4)$ \\
Sun & 20 & 28 \\
\hline
\end{tabular}

Note: the number of planet gears is in the parenthesis.

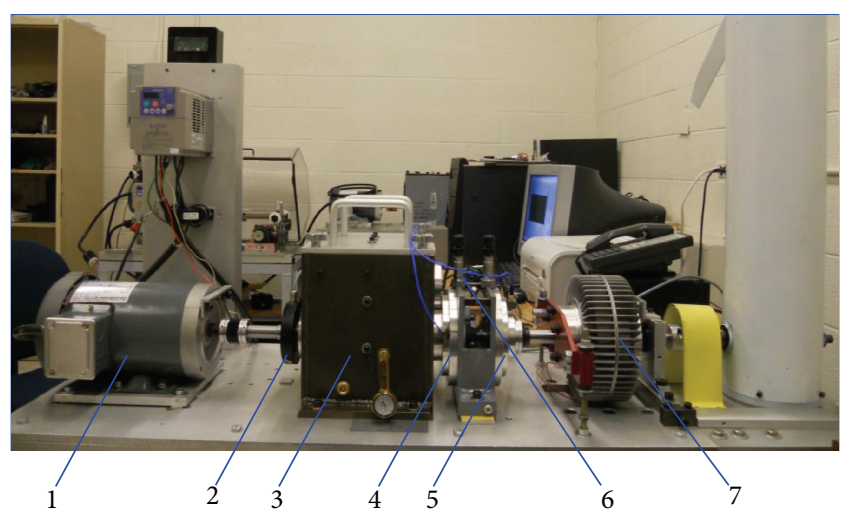

FIGURE 3: WT drivetrain test rig: (1) motor, (2) tachometer, (3) fixed-shaft gearbox, (4) planetary gearbox stage 1, (5) planetary gearbox stage 2, (6) accelerometers, and (7) loading device.

The drive motor speed decreases from $60 \mathrm{~Hz}$ to $50 \mathrm{~Hz}$, and the corresponding blade speed varies continuously from $0.486 \mathrm{~Hz}$ to $0.405 \mathrm{~Hz}$, within the typical rated speed range of wind turbine blades in real world. The motor speed is meanwhile recorded at a sampling frequency of $20 \mathrm{~Hz}$.

To simulate gear faults, two types of gear damage were introduced to sun gears of planetary gearbox stage 1 and stage 2, respectively, while other gears are healthy. Stage 1 sun gear has wear on every tooth and stage 2 sun gear has an individual chipped tooth, as illustrated in Figures 4(a) and 4(b). Therefore three sets of experiments are carried out as baseline, wear, and chipping.

Given the drive motor speed $f_{d}(t)$, we can calculate the characteristic frequencies of both stages of planetary gearbox according to the aforementioned gearbox configuration, as listed in Table 3. For example, at the motor speed $f_{d}(t)=$ $60 \mathrm{~Hz}$, the sun gear related characteristic frequencies are calculated as $f_{m 1}(t)=222.2 \mathrm{~Hz}, f_{s 1}^{(r)}(t)=13.3 \mathrm{~Hz}$, and $f_{s 1}(t)=44.4 \mathrm{~Hz}$ for stage 1 and $f_{m 2}(t)=48.6 \mathrm{~Hz}, f_{s 2}^{(r)}(t)=$ $2.2 \mathrm{~Hz}$, and $f_{s 1}(t)=6.9 \mathrm{~Hz}$ for stage 2 .

4.2. Baseline Signal Analysis. Firstly, we analyze the baseline signal for comparison study with the gear fault cases. In baseline case all gears are healthy. Figures 5(a) and 5(b) exhibit the waveform and Fourier spectrum of the baseline vibration signal, and Figure 5(c) shows the corresponding speed. The maximum meshing frequency is $222.2 \mathrm{~Hz}$ for stage 1 and $48.6 \mathrm{~Hz}$ for stage 2 . Since gear fault usually results in sidebands around meshing frequency and its harmonics, we focus on two frequency ranges of $0-400 \mathrm{~Hz}$ and $0-80 \mathrm{~Hz}$
TABLE 3: Planetary gearbox characteristic frequencies.

\begin{tabular}{lcc}
\hline Frequency & Stage 1 & Stage 2 \\
\hline Meshing $f_{m}(t)$ & $(100 / 27) f_{d}(t)$ & $(175 / 216) f_{d}(t)$ \\
Sun rotating $f_{s}^{(r)}(t)$ & $(2 / 9) f_{d}(t)$ & $(1 / 27) f_{d}(t)$ \\
Planet carrier rotating $f_{c}(t)$ & $(1 / 27) f_{d}(t)$ & $(7 / 864) f_{d}(t)$ \\
Sun fault $f_{s}(t)$ & $(20 / 27) f_{d}(t)$ & $(175 / 1512) f_{d}(t)$ \\
Planet fault $f_{p}(t)$ & $(5 / 54) f_{d}(t)$ & $(175 / 7776) f_{d}(t)$ \\
Ring fault $f_{r}(t)$ & $(4 / 27) f_{d}(t)$ & $(175 / 5400) f_{d}(t)$ \\
\hline
\end{tabular}

which cover $3 / 2$ times stage 1 and stage 2 meshing frequencies, respectively.

Figures 6(a) and 6(b) show the reassigned wavelet scalogram of the baseline signal. It clearly displays the constituent frequency components and their variations along with time. The value of each characteristic frequency at any time can be calculated according to the equations in Table 3; thus the instantaneous frequency curves in the time-frequency analysis results can be identified. In Figure 6(a), the gear meshing frequency minus the sun gear fault frequency $f_{m 1}(t)-f_{s 1}(t)$ and the gear meshing frequency minus four times the sun rotating frequency $f_{m 1}(t)-4 f_{s 1}^{(r)}(t)$ are presented, but they are not dominant in comparison with the other components almost uniformly distributed in higher frequency region $[200,400] \mathrm{Hz}$. Even for healthy gearboxes, manufacturing errors and minor defects are inevitable. Hence, the presence of these frequency components does not imply the sun gear fault. In Figure 6(b), only a few weak components are visible, showing that the gearbox is healthy, which is consistent with the experimental setting.

4.3. Detection of Sun Gear Wear. In this section we replaced the healthy stage 1 sun gear with a worn one while other settings are unchanged and detect the sun gear wear by comparing worn sun gear signal with baseline signal in joint time and frequency domain. The waveform, Fourier spectrum, and corresponding motor speed are plotted in Figures $7(a)-7(c)$. Since gear damage locates on stage 1 sun gear, in this case we focus on $0-400 \mathrm{~Hz}$ to look for fault symptom around stage 1 meshing frequency.

Figure 8 presents the reassigned wavelet scalogram of faulty sun signal. Compared to the baseline signal case in Figure 6(a), the gear meshing frequency minus the sun fault characteristic frequency $f_{m 1}(t)-f_{s 1}(t)$ and the gear meshing frequency minus four times the sun rotating frequency $f_{m 1}(t)-4 f_{s 1}^{(r)}(t)$ still remain. Besides, more characteristic frequencies appear, including the gear meshing frequency $f_{m 1}(t)$, the gear meshing frequency minus $7 / 4$ times the sun fault characteristic frequency $f_{m 1}(t)-(7 / 4) f_{s 1}(t)$, and the gear meshing frequency plus three times the sun fault characteristic frequency $f_{m 1}(t)+3 f_{s 1}(t)$. All these frequency components have a pronounced magnitude, and they are all related to the sun gear. This finding shows that the 1st stage sun gear is faulty, in consistence with the experimental setting. 


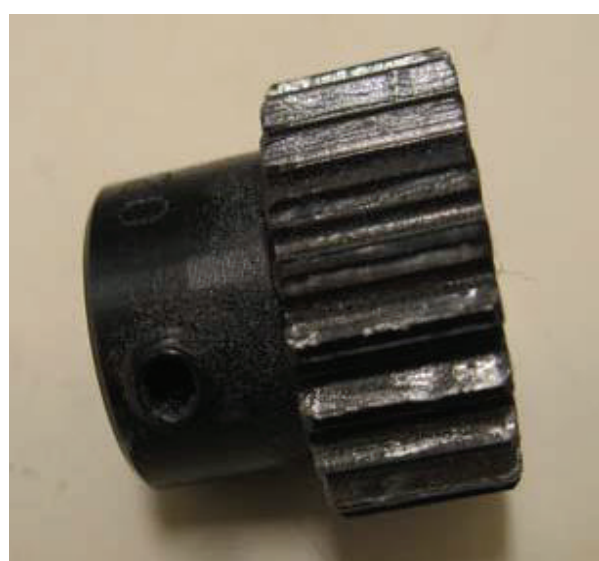

(a) Stage 1 sun gear wear

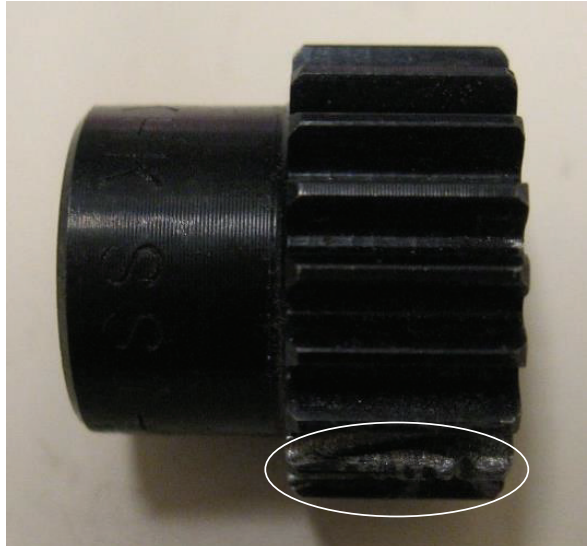

(b) Stage 2 sun gear chipping

FIGURE 4: Sun gear damage.

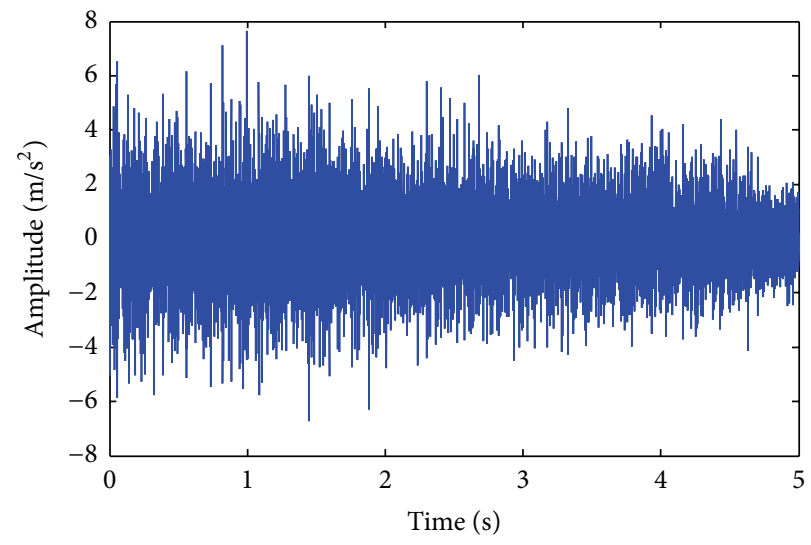

(a) Waveform

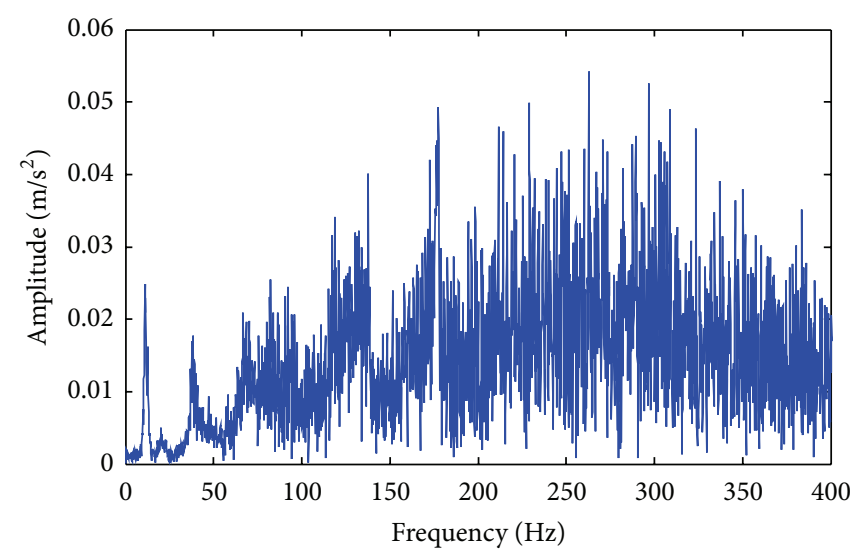

(b) Fourier spectrum

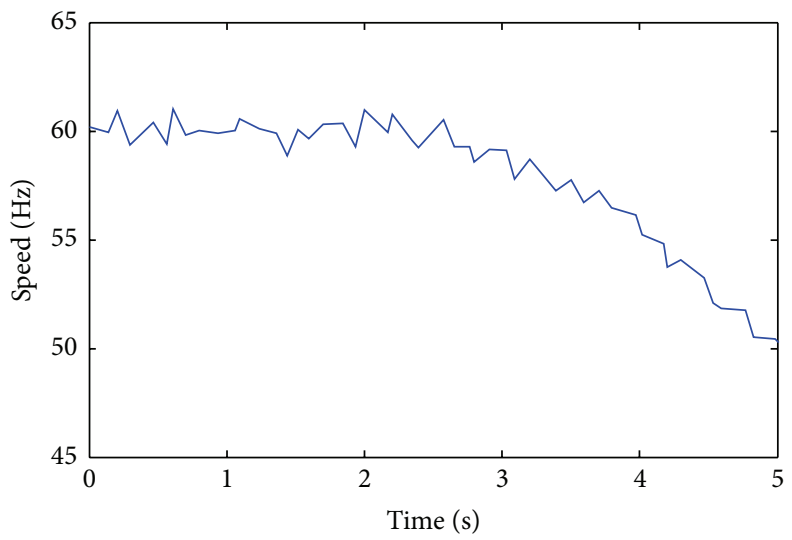

(c) Motor speed

FIGURE 5: Baseline signal.

4.4. Detection of Sun Gear Chipping. Another type of gear fault, that is, gear chipping, is brought in by replacing the healthy stage 2 sun gear with a chipped one while other settings remain the same with baseline case. Still we detect the fault by comparing chipped sun gear signal with baseline signal in joint time and frequency domain, but another frequency range of $0-80 \mathrm{~Hz}$ is focused on since the maximum stage 2 meshing frequency is $48.6 \mathrm{~Hz}$. The waveform, Fourier spectrum, and corresponding motor speed of chipped sun gear signal are presented in Figures 9(a)-9(c). 


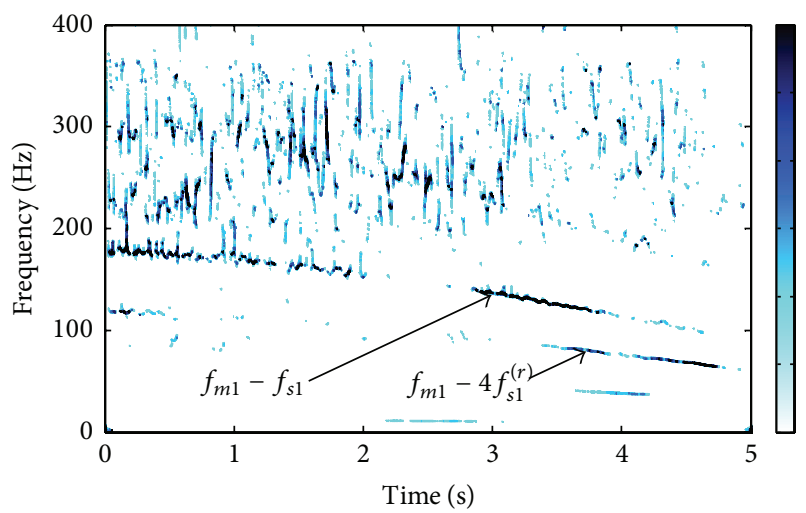

(a) Frequency band of $0-400 \mathrm{~Hz}$

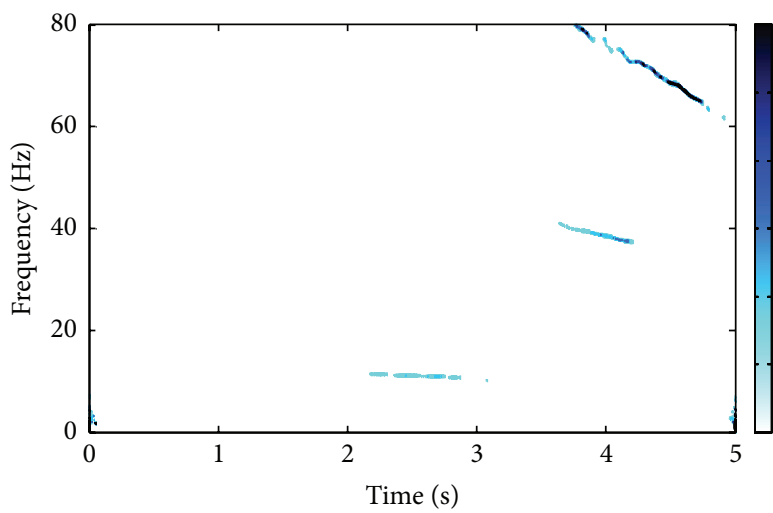

(b) Frequency band of $0-80 \mathrm{~Hz}$

FIGURE 6: Reassigned scalogram of baseline signal.

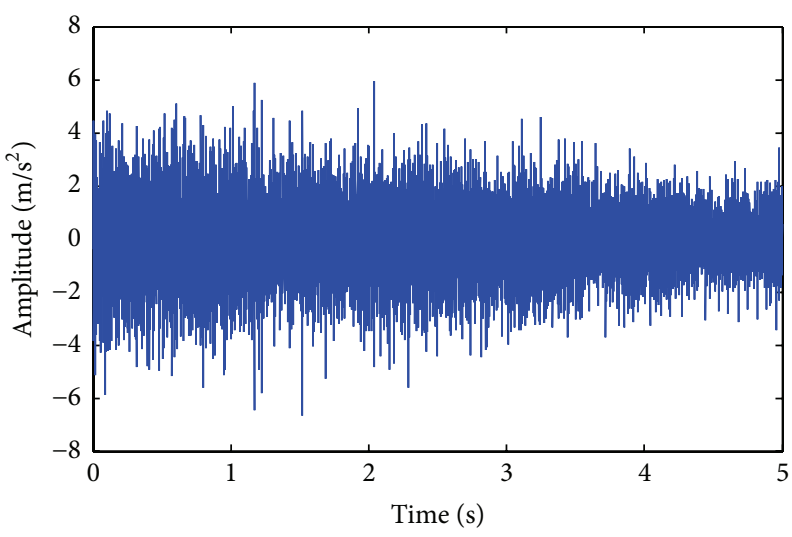

(a) Waveform

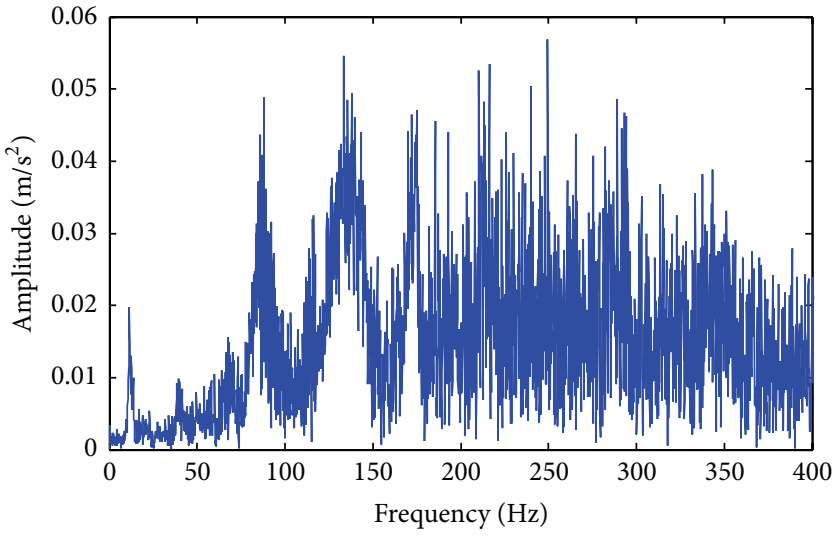

(b) Fourier spectrum

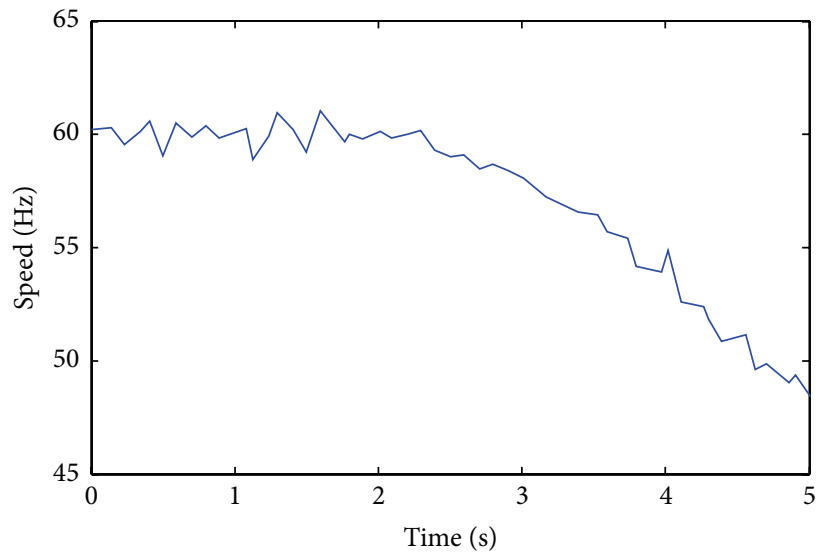

(c) Motor speed

FIGURE 7: Sun gear wear signal.

In the reassigned scalogram, Figure 10, except for the motor rotating frequency component $f_{d}$, two new characteristic frequency components are also evident. One corresponds to the meshing frequency minus the third harmonic of the sum of the sun gear fault characteristic frequency and the sun gear rotating frequency of the 2nd stage, $f_{m 2}-3\left[f_{s 2}^{(r)}+\right.$ $\left.f_{s 2}\right]$. The other is very close to motor rotating frequency, but by virtue of the fine time-frequency resolution, it is identified to be the sum of the meshing frequency and the second harmonic of sun gear fault characteristic frequency of the 2 nd stage, $f_{m 2}+2 f_{s 2}$ (as labeled with a black dashed line). These two extra frequency components correlate to the 2nd stage sun gear fault characteristic frequency and have prominent magnitude, thus implying the 2 nd stage sun gear fault. 


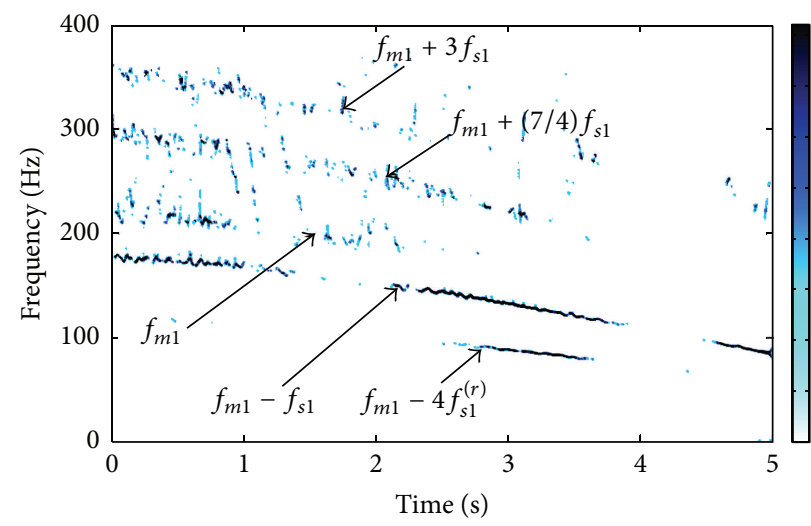

FIGURE 8: Reassigned scalogram of sun gear wear signal.

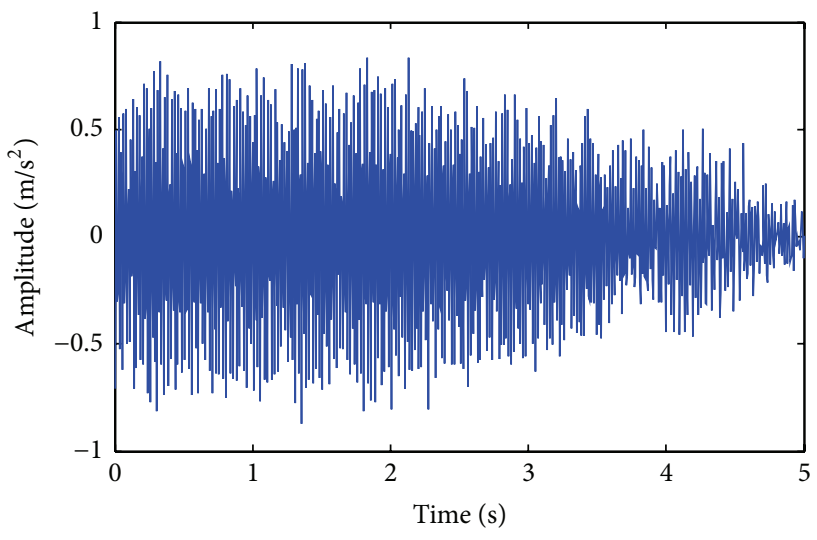

(a) Waveform

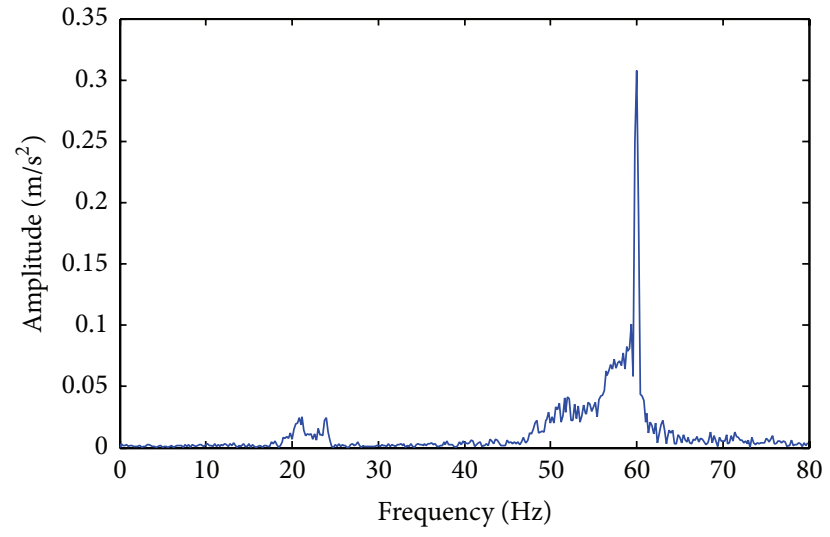

(b) Fourier spectrum

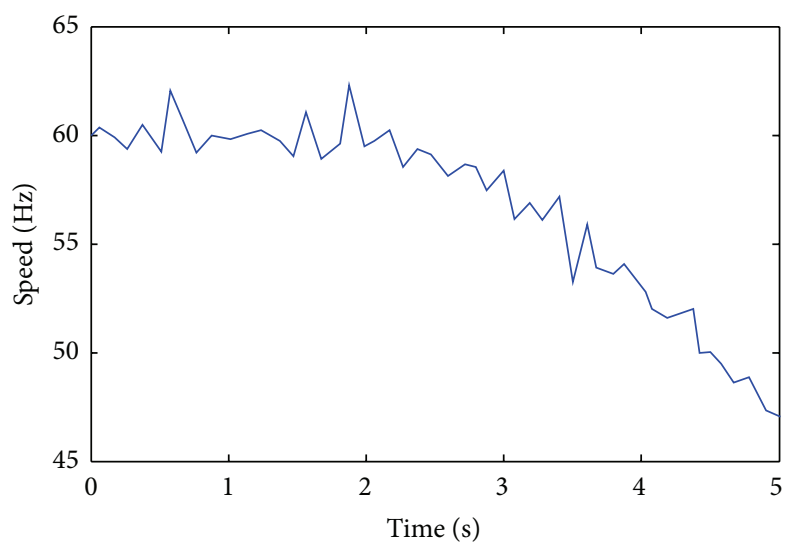

(c) Motor speed

FIgURE 9: Chipped sun gear signal.

The sun gear fault usually exhibits weak symptom in the vibration signal, because the sun gear is relatively far from the accelerometer mounted on the gearbox casing. But the time-varying sun gear fault characteristic frequencies are still identified, owing to the fine time-frequency resolution and capability in suppressing cross-term interferences of reassigned scalogram. This shows its effectiveness in extracting planetary gearbox fault features under nonstationary conditions.

\section{In Situ Signal Analysis}

In this section, we analyze the in situ collected signals of a wind turbine planetary gearbox in a wind farm, to further validate the reassigned scalogram for engineering applications.

5.1. Gearbox Configuration. The wind turbine drivetrain in a wind farm to be analyzed is composed of a one-stage 


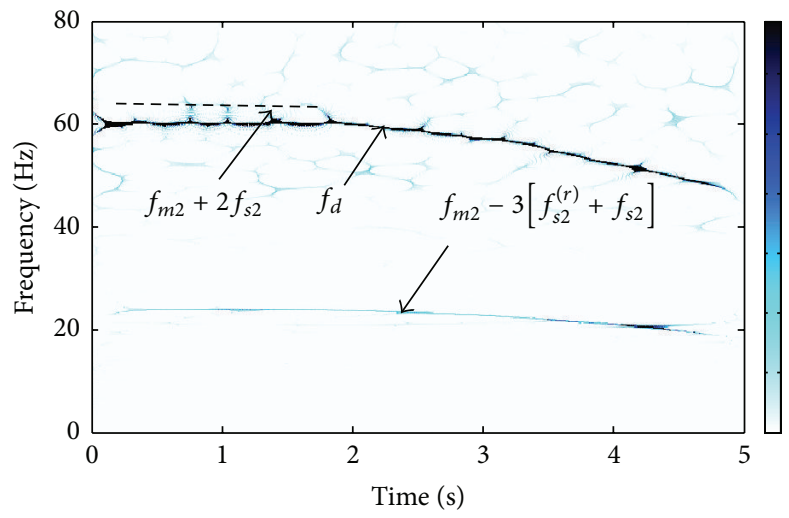

FIGURE 10: Reassigned scalogram of sun gear chipping signal.

TABLE 4: Planetary gearbox configuration parameters.

\begin{tabular}{lc}
\hline Gear & Number of gear teeth \\
\hline Ring & 96 \\
Planet & $37(3)$ \\
Sun & 21 \\
\hline
\end{tabular}

Note: the number of planet gears is in the parenthesis.

TABLE 5: Fixed-shaft gearbox configuration parameters.

\begin{tabular}{lcc}
\hline \multirow{2}{*}{ Gear } & \multicolumn{2}{c}{ Number of gear teeth } \\
& Input stage & Output stage \\
\hline Input gear & 71 & - \\
Intermediate shaft gear & 19 & 89 \\
Output gear & - & 30 \\
\hline
\end{tabular}

planetary gearbox and a two-stage fixed-shaft gearbox. The wind power flow path is as follows: blades $\rightarrow$ main shaft $\rightarrow$ planetary gearbox $\rightarrow$ fixed-shaft gearbox $\rightarrow$ generator. Considering the demand of wind farm information protection, photos of the wind turbine drivetrain are not attached in the paper. Tables 4 and 5 list the configuration parameters of the fixed-shaft gearbox and the planetary gearbox, respectively.

The rated rotating speed of the wind turbine blades is $25 \mathrm{rpm}$, that is, $0.416 \mathrm{~Hz}$. At the rated speed, the characteristic frequencies of the drivetrain gearboxes are calculated as listed in Table 6. They will be used to estimate the frequency components of vibration signals, for the wind turbine speed usually fluctuates around the rated speed.

The vibration signals from an accelerometer mounted on the planetary gearbox casing of both a normal and a faulty wind turbine are collected. Since the gear fault always results in abnormal sidebands or impulses centered around gear meshing frequency and its harmonics, a frequency band of $0-70 \mathrm{~Hz}$, which covers over $3 / 2$ times the planetary gearbox meshing frequency $(42.03 \mathrm{~Hz})$, will be focused on.

\subsection{Signal Analysis}

5.2.1. Normal Case. The waveform and Fourier spectrum of the normal wind turbine planetary gearbox vibration signal are plotted in Figures 11(a) and 11(b). Nevertheless, details of nonstationary characteristics are still veiled due to information loss. Figure 12 shows the reassigned wavelet scalogram of the normal wind turbine planetary gearbox vibration signal. The generator rotating frequency $27 \mathrm{~Hz}$ and the planetary gearbox meshing frequency $42 \mathrm{~Hz}$ are dominant. The second harmonic of generator rotating frequency $54 \mathrm{~Hz}$ is also visible, but in a relatively low magnitude. All these frequencies are time-varying due to the unsteady speed of wind turbine blades caused by the nonstationary wind power. Thus it is difficult to identify frequency components via traditional Fourier transform based spectral analysis.

5.2.2. Faulty Planet Gear Case. In the faulty case, similarly the waveform and Fourier spectrum of faulty planetary gearbox vibration signal are presented in Figures 13(a) and 13(b). Due to the unsteady motor speed and nonstationary conditions, it is difficult to identify frequency components via traditional spectral analysis. Figure 14 shows the reassigned scalogram of the faulty wind turbine planetary gearbox vibration signal. It has a time-frequency structure far different from that in the normal case. Only the planetary gearbox meshing frequency is dominant. In addition, some big impulses appear almost periodically, as labeled by evenly spaced vertical lines with capitals A-G in Figure 14(a). The interval between adjacent impulses corresponds approximately to the planet carrier rotating frequency $0.44 \mathrm{~Hz}$. Furthermore, if we zoom in Figure 14(a) and focus on the interval between two consecutive primary impulses, for example, B and C, some small almost periodic impulses also appear, as indicated by lower-case letters a-f in Figure 14(b). The interval between these adjacent small impulses corresponds approximately to the planet gear fault characteristic frequency, that is, $2.27 \mathrm{~Hz}$. These findings imply the planet gear fault, in accordance with the check result after opening the planetary gearbox.

The planet gear fault symptom is complicated, because the planet gear not only spins around its own axis but also revolves around the sun gear with the planet carrier, and meanwhile it meshes with both the sun gear and the ring gear. But the gear fault induced impulses are still identified, illustrating the effectiveness of reassigned scalogram in extracting complicated fault features under nonstationary conditions. 
TABLE 6: Characteristic frequencies of drivetrain gearbox.

\begin{tabular}{cccccccccc}
\hline & \multicolumn{2}{c}{ Planetary } & \multicolumn{3}{c}{ Fixed-shaft stage 1 } & \multicolumn{3}{c}{ Fixed-shaft stage 2 } \\
Mesh & Ring & Planet & Sun & Mesh & Drive & Driven & Mesh & Drive & Driven \\
\hline 42.03 & 0.44 & 2.27 & 2.01 & 173.18 & 2.44 & 9.11 & 811.20 & 9.11 & 27.04 \\
\hline
\end{tabular}

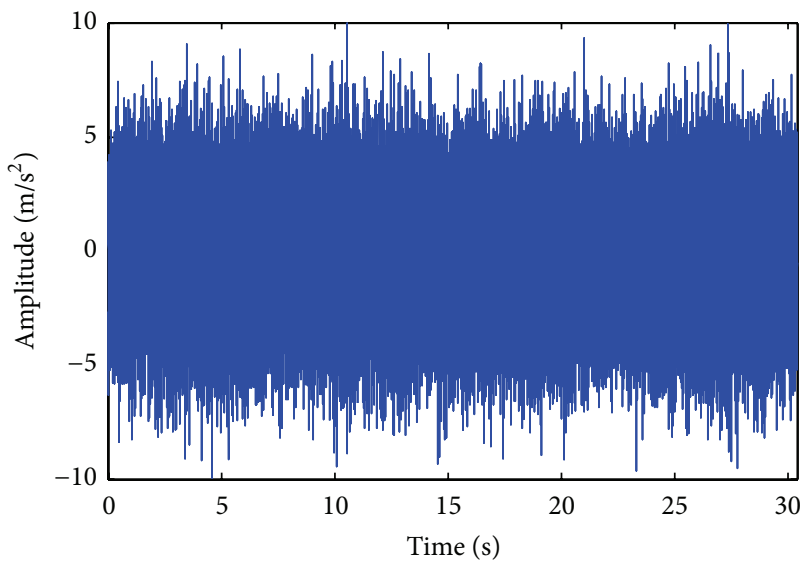

(a) Waveform

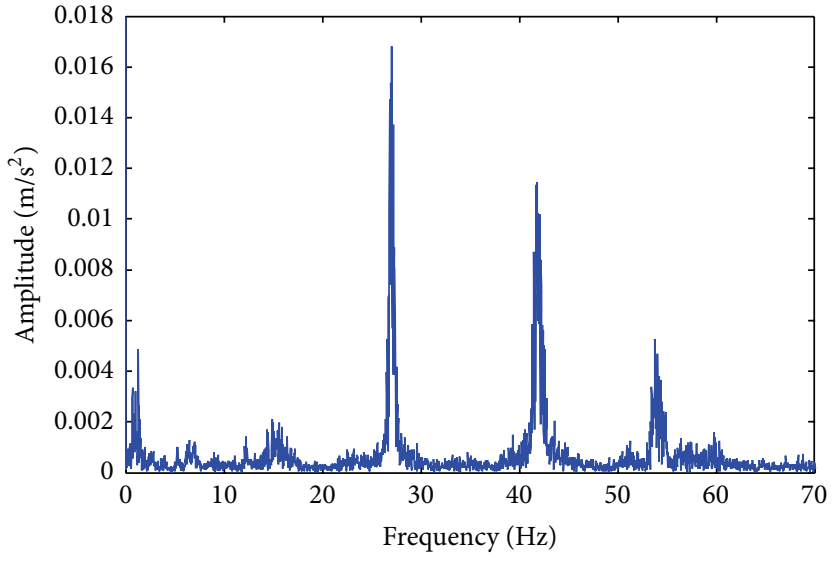

(b) Fourier spectrum

Figure 11: Normal signal.

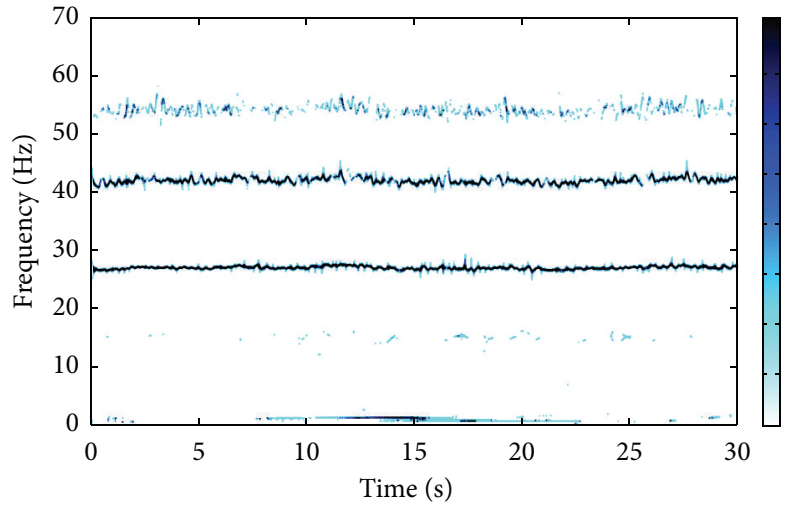

FIGURE 12: Reassigned scalogram of normal signal.

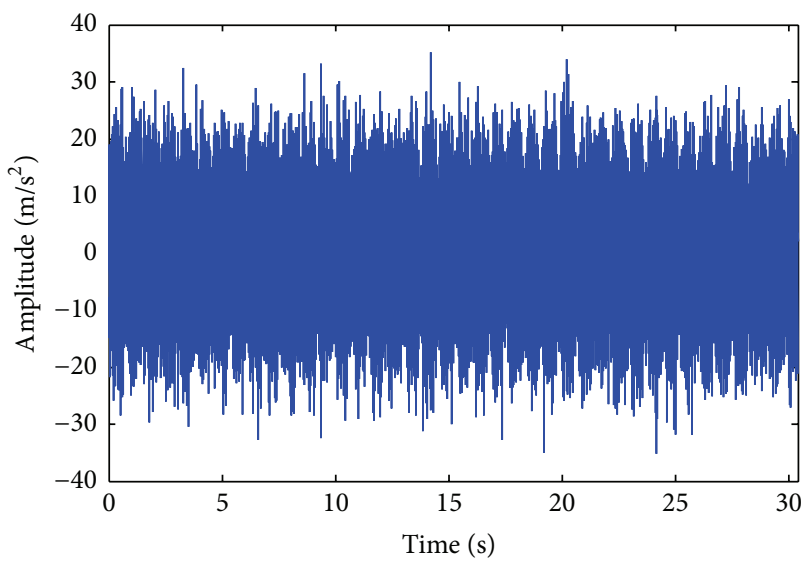

(a) Waveform

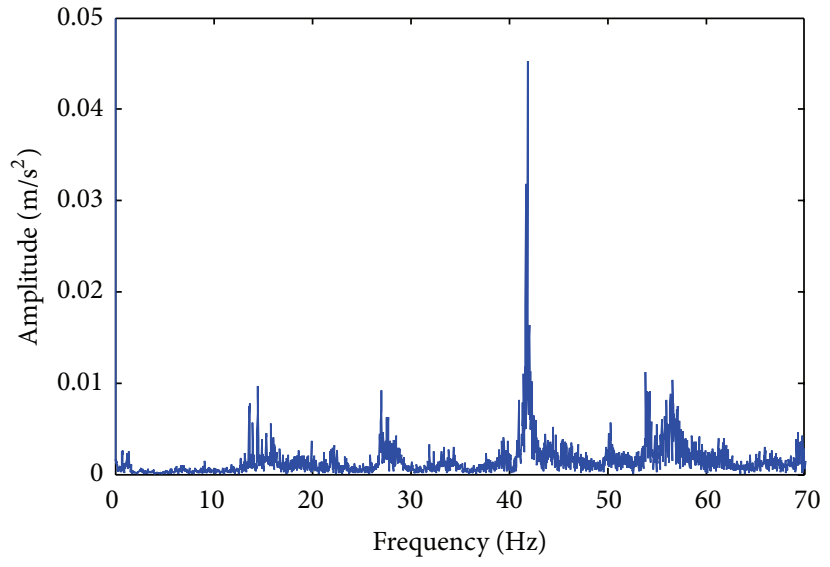

(b) Fourier spectrum

FIGURE 13: Faulty signal. 


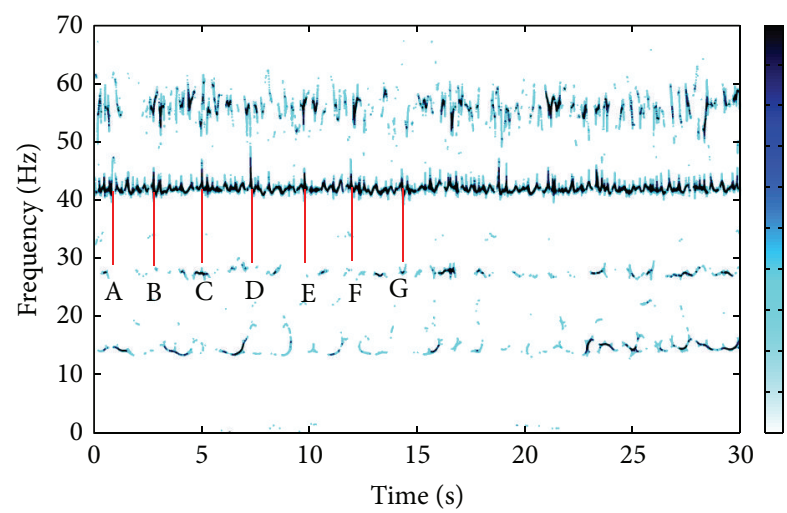

(a) Full time span view

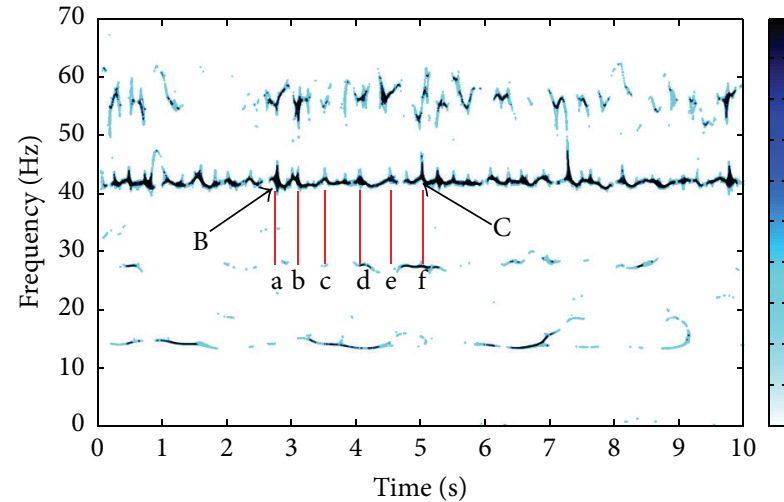

(b) Close-up view of the interval from 0 to $10 \mathrm{~s}$

FIGURE 14: Reassigned scalogram of faulty signal.

\section{Conclusions}

The reassigned wavelet scalogram has excellent merits of fine time-frequency resolution and capability in suppressing cross-term interferences and thus is suited to extract the time-frequency features of nonstationary signals. We have validated the effectiveness of this method for planetary gearbox fault diagnosis under nonstationary conditions, using both lab experimental signals and in situ measured datasets. Both the time-varying gear characteristic frequencies and gear fault induced impulses are identified, and thereby the localized and distributed sun gear fault, as well as naturally developed planet gear fault, are diagnosed, even though they have weak and complicated fault symptoms under nonstationary conditions.

\section{Conflict of Interests}

The authors declare that there is no conflict of interests regarding the publication of this paper.

\section{Acknowledgments}

This work is supported by the National Natural Science Foundation of China (11272047 and 51475038), the Program for New Century Excellent Talents in University (NCET-120775), and the Natural Sciences and Engineering Research Council of Canada.

\section{References}

[1] P. D. Samuel and D. J. Pines, "A review of vibration-based techniques for helicopter transmission diagnostics," Journal of Sound and Vibration, vol. 282, no. 1-2, pp. 475-508, 2005.

[2] Y. Guo and R. G. Parker, "Analytical determination of mesh phase relations in general compound planetary gears," Mechanism and Machine Theory, vol. 46, no. 12, pp. 1869-1887, 2011.

[3] Y. Lei, J. Lin, M. J. Zuo, and Z. He, "Condition monitoring and fault diagnosis of planetary gearboxes: a review," Measurement, vol. 48, no. 1, pp. 292-305, 2014.
[4] P. D. McFadden, "Window functions for the calculation of the time domain averages of the vibration of the individual planet gears and sun gear in an epicyclic gearbox," Journal of Vibration and Acoustics, Transactions of the ASME, vol. 116, no. 2, pp. 179187, 1994.

[5] M. Inalpolat and A. Kahraman, "A theoretical and experimental investigation of modulation sidebands of planetary gear sets," Journal of Sound and Vibration, vol. 323, no. 3-5, pp. 677-696, 2009.

[6] M. Inalpolat and A. Kahraman, "A dynamic model to predict modulation sidebands of a planetary gear set having manufacturing errors," Journal of Sound and Vibration, vol. 329, no. 4, pp. 371-393, 2010.

[7] Z. Feng and M. J. Zuo, "Vibration signal models for fault diagnosis of planetary gearboxes," Journal of Sound and Vibration, vol. 331, no. 22, pp. 4919-4939, 2012.

[8] J. McNames, "Fourier series analysis of epicyclic gearbox vibration," Journal of Vibration and Acoustics, vol. 124, no. 1, pp. 150$160,2002$.

[9] J. Chen, C. Zhang, X. Zhang, Y. Zi, S. He, and Z. Yang, "Planetary gearbox condition monitoring of ship-based satellite communication antennas using ensemble multiwavelet analysis method," Mechanical Systems and Signal Processing, vol. 54-55, pp. 277-292, 2015.

[10] Y. Lei, D. Han, J. Lin, and Z. He, "Planetary gearbox fault diagnosis using an adaptive stochastic resonance method," Mechanical Systems and Signal Processing, vol. 38, no. 1, pp. 113124, 2013.

[11] Z. Feng, M. Liang, Y. Zhang, and S. Hou, "Fault diagnosis for wind turbine planetary gearboxes via demodulation analysis based on ensemble empirical mode decomposition and energy separation," Renewable Energy, vol. 47, pp. 112-126, 2012.

[12] T. Barszcz and R. B. Randall, "Application of spectral kurtosis for detection of a tooth crack in the planetary gear of a wind turbine," Mechanical Systems and Signal Processing, vol. 23, no. 4, pp. 1352-1365, 2009.

[13] H. Sun, Y. Zi, Z. He, J. Yuan, X. Wang, and L. Chen, “Customized multiwavelets for planetary gearbox fault detection based on vibration sensor signals," Sensors, vol. 13, no. 1, pp. 1183-1209, 2013.

[14] H. Li, J. Zhao, and W. Song, "An offline fault diagnosis method for planetary gearbox based on empirical mode decomposition 
and adaptive multi-scale morphological gradient filter," Journal of Vibroengineering, vol. 17, pp. 705-719, 2015.

[15] Z. Feng, F. Chu, and M. J. Zuo, "Time-frequency analysis of time-varying modulated signals based on improved energy separation by iterative generalized demodulation," Journal of Sound and Vibration, vol. 330, no. 6, pp. 1225-1243, 2011.

[16] Z. Feng, X. Chen, M. Liang, and F. Ma, "Time-frequency demodulation analysis based on iterative generalized demodulation for fault diagnosis of planetary gearbox under nonstationary conditions," Mechanical Systems and Signal Processing, vol. 62-63, pp. 54-74, 2015.

[17] Z. Feng and M. Liang, "Fault diagnosis of wind turbine planetary gearbox under nonstationary conditions via adaptive optimal kernel time-frequency analysis," Renewable Energy, vol. 66, pp. 468-477, 2014.

[18] W. Bartelmus and R. Zimroz, "Vibration condition monitoring of planetary gearbox under varying external load," Mechanical Systems and Signal Processing, vol. 23, no. 1, pp. 246-257, 2009.

[19] F. Chaari, M. S. Abbes, F. V. Rueda, A. F. del Rincon, and M. Haddar, "Analysis of planetary gear transmission in nonstationary operations," Frontiers of Mechanical Engineering, vol. 8, no. 1, pp. 88-94, 2013.

[20] J. Yang and L. Zhang, "Dynamic response and dynamic load of wind turbine planetary gear transmission system under changing excitation," Applied Mechanics and Materials, vol. 121126, pp. 2671-2675, 2011.

[21] Z. Feng, M. Liang, and F. Chu, "Recent advances in timefrequency analysis methods for machinery fault diagnosis: a review with application examples," Mechanical Systems and Signal Processing, vol. 38, no. 1, pp. 165-205, 2013.

[22] O. Rioul and P. Flandrin, "Time-scale energy distributions: a general class extending wavelet transforms," IEEE Transactions on Signal Processing, vol. 40, no. 7, pp. 1746-1757, 1992.

[23] F. Hlawatsch and G. F. Boudreaux-Bartels, "Linear and quadratic time-frequency signal representations," IEEE Signal Processing Magazine, vol. 9, no. 2, pp. 21-67, 1992.

[24] L. Cohen, “Time-frequency distributions: a review," Proceedings of the IEEE, vol. 77, no. 7, pp. 941-981, 1989.

[25] Z. K. Peng, F. L. Chu, and P. W. Tse, "Detection of the rubbingcaused impacts for rotor-stator fault diagnosis using reassigned scalogram," Mechanical Systems and Signal Processing, vol. 19, no. 2, pp. 391-409, 2005.

[26] H. Ma, T. Yu, Q. Han, Y. Zhang, B. Wen, and C. Xuelian, "Timefrequency features of two types of coupled rub-impact faults in rotor systems," Journal of Sound and Vibration, vol. 321, no. 3-5, pp. 1109-1128, 2009.

[27] X. Chen, Z. Feng, and M. Liang, "Fault feature extraction of planetary gearboxes under nonstationary conditions based on reassigned wavelet scalogram," in Proceedings of the IEEE International Instrumentation and Measurement Technology Conference, pp. 294-299, Pisa, Italy, May 2015.

[28] F. Auger and P. Flandrin, "Improving the readability of timefrequency and time-scale representations by the reassignment method," IEEE Transactions on Signal Processing, vol. 43, no. 5, pp. 1068-1089, 1995. 


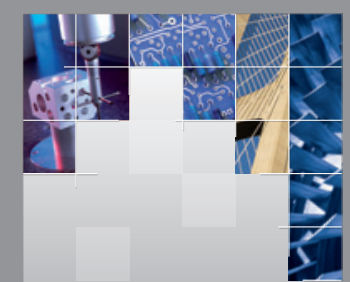

\section{Enfincering}
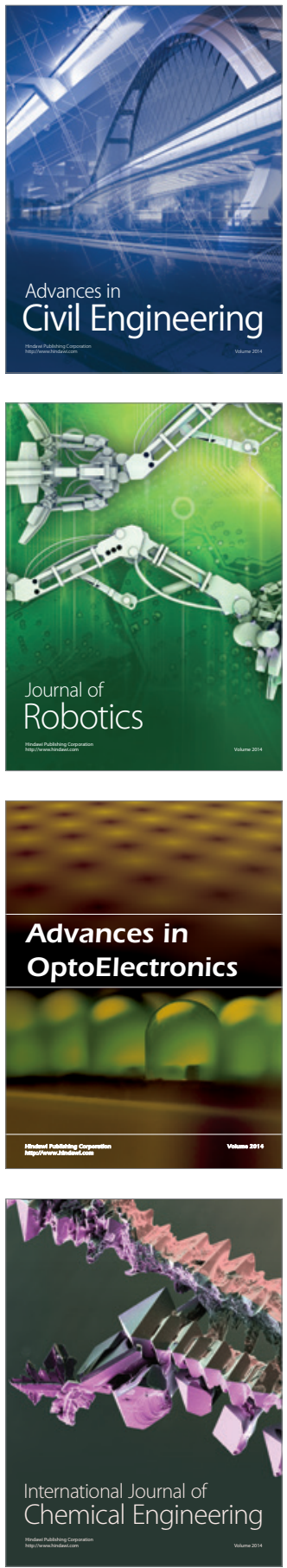

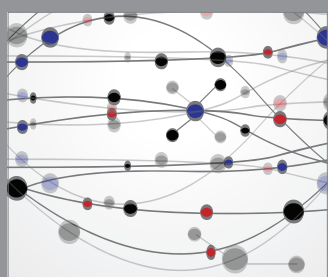

The Scientific World Journal

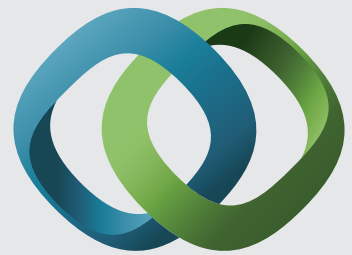

\section{Hindawi}

Submit your manuscripts at

http://www.hindawi.com
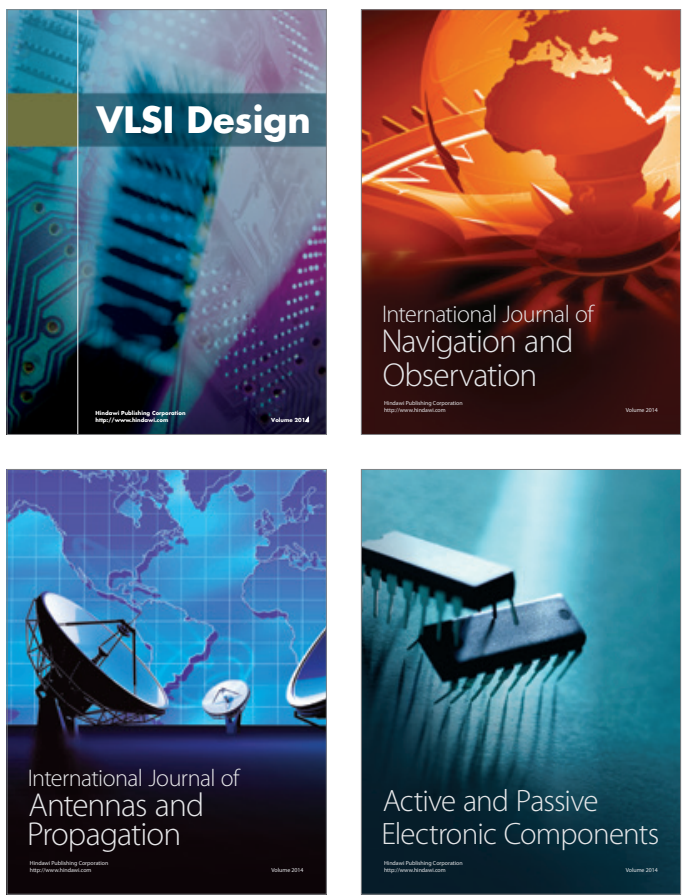
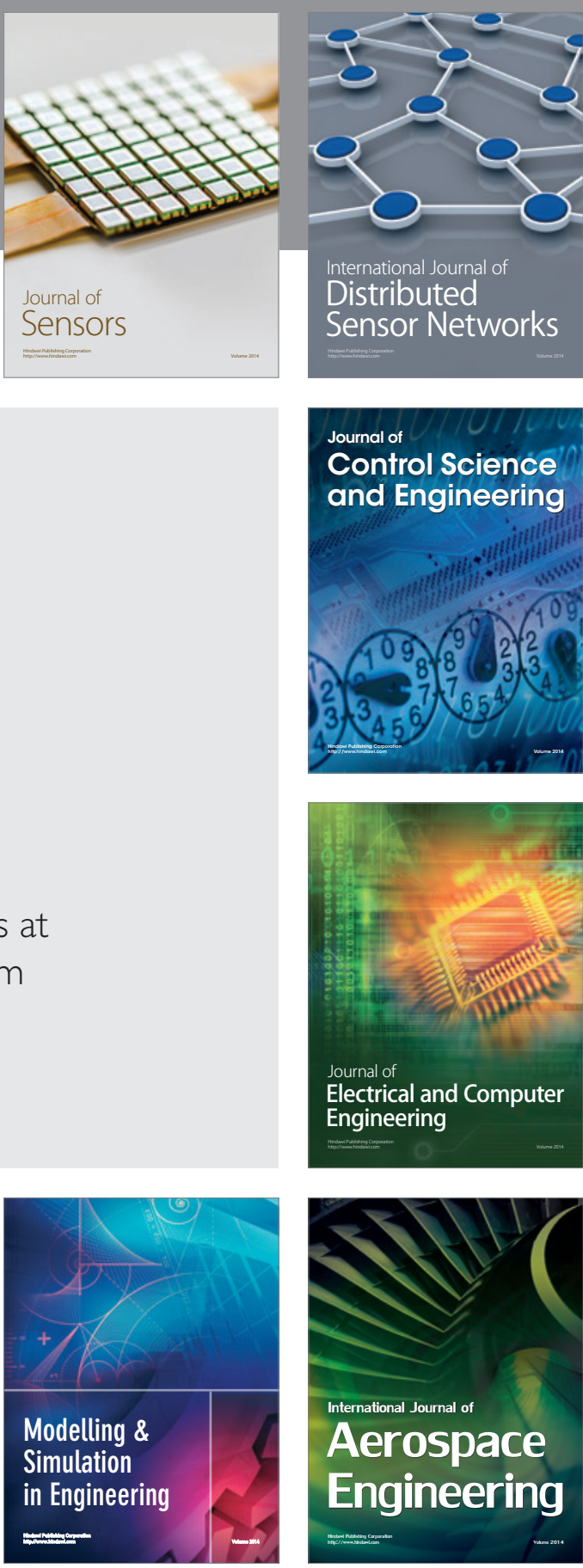

International Journal of

Distributed

Sensor Networks

Journal of

Control Science

and Engineering
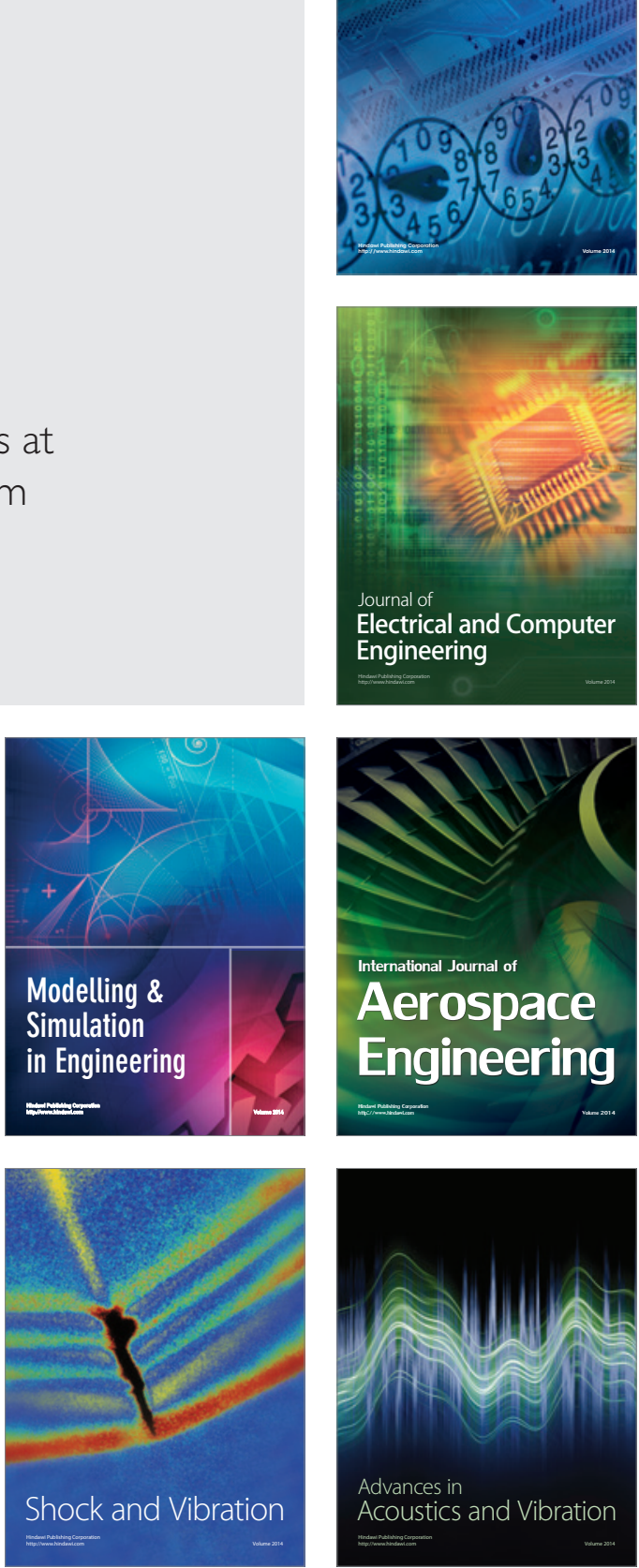